\title{
Widespread disruptive selection in the wild is associated with intense resource competition
}

\author{
Ryan A Martin ${ }^{1,2^{*}}$ and David W Pfennig
}

\begin{abstract}
Background: Disruptive selection has been documented in a growing number of natural populations. Yet, its prevalence within individual systems remains unclear. Furthermore, few studies have sought to identify the ecological factors that promote disruptive selection in the wild. To address these issues, we surveyed 15 populations of Mexican spadefoot toad tadpoles, Spea multiplicata, and measured the prevalence of disruptive selection acting on resource-use phenotypes. We also evaluated the relationship between the strength of disruptive selection and the intensity of intraspecific competition —an ecological agent hypothesized to be an important driver of disruptive selection.

Results: Disruptive selection was the predominant mode of quadratic selection across all populations. However, a directional component of selection favoring an extreme ecomorph - a distinctive carnivore morph — was also common. Disruptive selection was strongest in populations experiencing the most intense intraspecific competition, whereas stabilizing selection was only found in populations experiencing relatively weak intraspecific competition.
\end{abstract}

Conclusions: Disruptive selection can be common in natural populations. Intraspecific competition for resources may be a key driver of such selection.

Keywords: Competition, Disruptive selection, Resource polymorphism, Selection differential, Spadefoot toad, Quadratic selection

\section{Background}

Disruptive selection occurs in a population when two or more modal phenotypes have higher fitness than the intermediate phenotypes between them [1]. Disruptive selection has long been viewed as important in maintaining and increasing variation within natural populations [1-3]; favoring the evolution of alternative phenotypes $[4,5]$ and sexual dimorphism [6-8]; and even initiating speciation [6,8-12]. Nevertheless, compared to the other main modes of selection-directional selection and stabilizing selection-disruptive selection has traditionally received much less attention.

Despite this relative lack of attention given to disruptive selection, recent meta-analyses suggest that disruptive selection may be at least as common as stabilizing selection [13,14]. Indeed, an increasing number of

\footnotetext{
* Correspondence: ryan_martin@ncsu.edu
'Department of Biology, CB\#3280, University of North Carolina, Chapel Hill,

'Department of Biology, CB\#3280, University of North Carolina, Chapel Hill, NC 27599, USA

${ }^{2}$ Present address: Department of Biology, CB\#7617, North Carolina State University, Raleigh, NC 27695, USA
}

(c) 2012 Martin and Pfennig; licensee BioMed Central Ltd. This is an Open Access article distributed under the terms of the Creative Commons Attribution License (http://creativecommons.org/licenses/by/2.0), which permits unrestricted use, distribution, and reproduction in any medium, provided the original work is properly cited. populations [15-24]. Yet, although these data imply that disruptive selection may be widespread, we still do not know how prevalent it can be within individual systems where it has been found to occur [22].

Additionally, the actual causes of disruptive selection have been relatively understudied. Longstanding theory suggests that disruptive selection arises from negative frequency-dependent interactions, such as those stemming from intraspecific competition, predation, mutualism, and parasitism [10,25-28]. To illustrate how intraspecific competition generates disruptive selection $[3,8,10,29,30]$, consider first that-owing to functional trade-offs [17-20,31-35] - individuals with certain phenotypes are generally better adapted than are individuals with other phenotypes at utilizing specific resource types [36]. Yet, as any one of these modal phenotypes becomes common, it will tend to suffer from resource depletion, as these individuals compete more against themselves than against other resource-use phenotypes in the same population [18,37]. Consequently, the less common 
modal phenotype(s) will be favored, because they will experience reduced competition for their resources $[10,35,38]$. In this way, intraspecific competition acts as an agent of frequency dependent disruptive selection, which favors two or more modal resource-use phenotypes in the same population $[35,38,39]$.

Empirical studies largely support this theory. Indeed, intraspecific competition has been shown to cause disruptive selection in natural populations of three-spine sticklebacks [22,35], spadefoot toad tadpoles [18,21], and Eurasian perch [23], and it has been implicated as causing disruptive selection in various other systems [15$17,19,20,40]$. Moreover, several studies have shown that competition generates negative frequency-dependence among different resource-use phenotypes [31,41-44], which (as noted above) is a hallmark of competitively mediated disruptive selection [38].

Nevertheless, relatively few comprehensive surveys of natural populations have sought to identify the ecological conditions that are associated with disruptive selection [22,23]. For example, the relationship between spatial variation in the strength of intraspecific resource competition and the occurrence and intensity of disruptive selection has been little examined in unmanipulated wild populations. This relationship has likely been difficult to evaluate because doing so requires evaluating selection in replicated populations, some of which experience weak resource competition and some of which experience strong resource competition.

We sought to fill these gaps in our knowledge concerning the prevalence and correlates of disruptive selection in natural populations. We specifically tested two predictions: first, that disruptive selection may be common within certain systems, and second, that disruptive selection would be more intense under conditions of greater resource competition. We tested these two predictions in natural populations of Mexican spadefoot toads (Spea multiplicata).

Spadefoot toad tadpoles are well-suited for such studies, because they express a remarkable range of trophic phenotypes in the wild $[45,46]$. The extremes of this variation are represented by two ecomorphs that comprise a resource polymorphism: an "omnivore" morpha round-bodied tadpole with a long intestine, small jaw muscles, numerous rows of keratinized denticles, and smooth keratinized mouthparts that feeds primarily on the pond bottom, and a "carnivore" morph-a narrowbodied tadpole with a short intestine, greatly enlarged jaw muscles, few rows of keratinized denticles, and notched, serrated keratinized mouthparts that feeds mostly in the water column $[18,47,48]$. Omnivores are generalists, which feed mostly on microscopic detritus, algae, and small crustaceans, whereas carnivores are specialists, which feed mostly on anostracan fairy shrimp $[37,49]$.
Although carnivore development is induced by consumption of fairy shrimp [50], heritable variation for morph development exists within natural populations [48,51]. The degree to which a population expresses this resource polymorphism varies across ponds, and is, in part, associated with variation in conspecific density and ecological opportunity (i.e., the presence of underutilized, accessible resources). Specifically, bimodality in trophic phenotypes is greatest in ponds where conspecific density and ecological opportunity are highest [46].

Previous research suggests that intermediate phenotypes are disfavored by disruptive selection in this system $[18,21,46]$. In particular, compared to tadpoles with intermediate phenotypes, omnivores and carnivores are larger, more developmentally advanced, and more likely to survive to metamorphosis [18,21]. Furthermore, previous experiments have shown that this disruptive selection reflects negative frequency-dependent interactions driven by ecological specialization and resource competition $[43,46]$.

However, it is unclear how prevalent disruptive selection is among populations of S. multiplicata or how ecological variation impacts the mode and strength of selection in this system. We therefore addressed these issues by measuring the mode and magnitude of phenotypic selection, as well as the strength of intraspecific competition within natural populations. We focused on populations of Mexican spadefoot toad tadpoles (S. multiplicata) in the San Simon Valley of southeastern Arizona and southwestern New Mexico, USA (Figure 1). These populations are ideal for addressing the above issues because the $S$. multiplicata in these populations express a resource polymorphism that is thought to have arisen from disruptive selection $[18,21,46]$. Moreover, previous research suggests that disruptive selection may be widespread in these populations $[18,21]$. Finally, the $S$. multiplicata in these populations experience a wide array of ecological conditions over a small geographic area $[21,52,53]$.

We made a priori predictions based on theory as well as the prior work on this system (see above). Specifically, we predicted that disruptive selection on tadpole trophic morphology would be widespread in our surveyed populations. We also predicted that this disruptive selection would be strongest in ponds where intraspecific competition is the most intense (as measured by conspecific density and per capita resource abundance).

\section{Results}

\section{Evaluating the prevalence of disruptive selection}

Disruptive selection was the predominant mode of quadratic selection on tadpole trophic morphology (Figure 1). Indeed, disruptive selection, identified by a significantly positive $\gamma$ (Table 1) and a fitness minimum (Figure 2), occurred at least once in $73 \%$ of the unique ponds sampled (11 of 15 unique ponds) and in 59\% of our total 


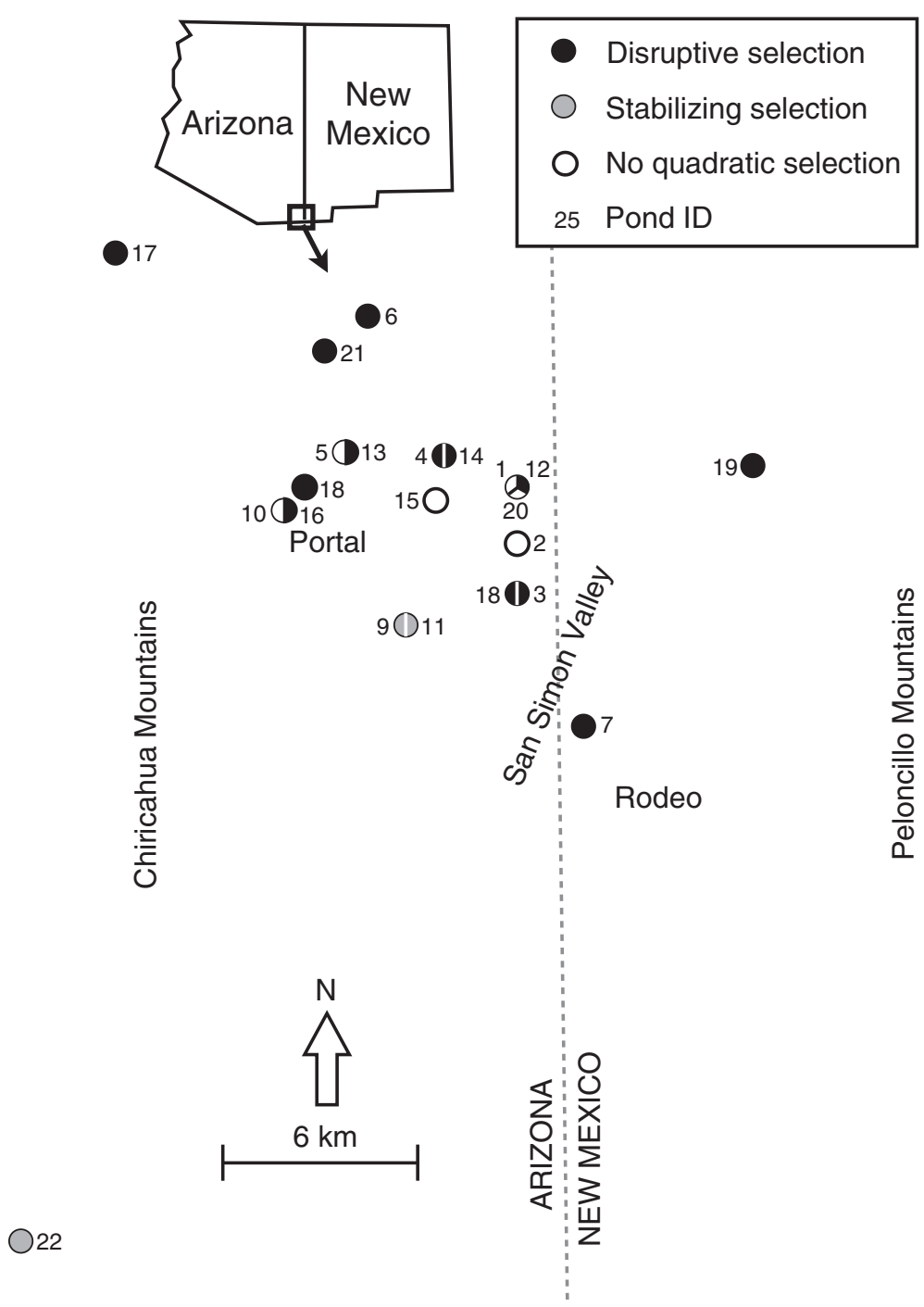

Figure 1 Localities. Map of study area illustrating locations of ponds sampled and the form of quadratic selection in each pond. Symbols for ponds sampled in multiple years are divided to show the form of quadratic selection in each year. The numbers beside each symbol section correspond to each collection's pond ID referenced as "Map ID" in Tables.

collections (Table 1; 13 of 22 collections). In contrast, stabilizing selection, identified by a significantly negative $\gamma$ (Table 1) and a fitness maximum (Figure 2), occurred in $13 \%$ of the unique ponds sampled ( 2 of 15 unique ponds) and in $14 \%$ of our total pond collections (Figure 1, Table 1; 3 of 22 collections). Additionally, a significant directional component of selection favoring carnivore-like phenotypes was present in $80 \%$ of the unique ponds sampled (Table 1 ; 12 of 15 unique ponds) and $82 \%$ of total pond collections (Table 1; 18 of 22 collections).

\section{Evaluating whether competition predicts the strength of disruptive selection}

As predicted, disruptive selection was more intense in ponds with greater intraspecific competition (i.e., lower per capita resource density; Figure $3 \mathrm{~A}, F_{1,20}=9.861$, $P=.005$, median regression coefficient from bootstrapping $=-.016,95 \%$ CI: $-.023--.011$, higher conspecific density; Figure $3 \mathrm{~B}, F_{1,20}=10.088, P=.004$, median regression coefficient from bootstrapping $=.02395 \% \mathrm{CI}$ : $.016-.030)$.

\section{Discussion}

Interest in disruptive selection arises from its potential role in maintaining and accentuating variation within populations (including polymorphism) and in promoting speciation [2]. Despite this longstanding interest, the prevalence of disruptive selection in natural populations, and therefore the relative importance of disruptive selection in generating biological diversity, remains unclear. 
Table 1 The mode and strength of selection on trophic morphology in natural ponds

\begin{tabular}{|c|c|c|c|c|c|c|c|}
\hline Pond & Map ID & Year & $\beta / \gamma$ & Selection differentials & $\mathrm{SE} / 2 \mathrm{SE}$ & $t$ & $P$ \\
\hline \multirow[t]{2}{*}{ AZ0602 } & 1 & 2006 & $\beta$ & .022 & .006 & 3.678 & .0003 \\
\hline & & & Y & .013 & .012 & 1.181 & .240 \\
\hline \multirow[t]{2}{*}{ AZ0603 } & 2 & 2006 & $\beta$ & .020 & .005 & 3.667 & .0003 \\
\hline & & & $\gamma$ & .019 & .011 & 1.639 & .103 \\
\hline \multirow[t]{2}{*}{ AZ0604 } & 3 & 2006 & $\beta$ & .014 & .008 & 1.622 & .111 \\
\hline & & & v & .039 & .013 & 2.874 & .006 \\
\hline \multirow[t]{2}{*}{ AZ0605 } & 4 & 2006 & $\beta$ & .031 & .009 & 3.487 & .0006 \\
\hline & & & $\gamma$ & .126 & .014 & 8.258 & $<.0001$ \\
\hline \multirow[t]{2}{*}{ AZ0606 } & 5 & 2006 & $\beta$ & .003 & .002 & 1.423 & .159 \\
\hline & & & $\gamma$ & -.001 & .004 & -.170 & .866 \\
\hline \multirow[t]{2}{*}{ AZ0607 } & 6 & 2006 & $\beta$ & -.017 & .007 & -2.354 & .020 \\
\hline & & & $\gamma$ & .038 & .012 & 2.920 & .004 \\
\hline \multirow[t]{2}{*}{ NM0608 } & 7 & 2006 & $\beta$ & .009 & .004 & 2.253 & .025 \\
\hline & & & Y & .024 & .006 & 3.596 & .0004 \\
\hline \multirow[t]{2}{*}{ AZ0710 } & 8 & 2007 & $\beta$ & .026 & .006 & 3.857 & .0002 \\
\hline & & & Y & .043 & .008 & 4.552 & $<.0001$ \\
\hline \multirow[t]{2}{*}{ AZ0711 } & 9 & 2007 & $\beta$ & .076 & .009 & 8.262 & $<.0001$ \\
\hline & & & $\gamma$ & -.060 & .018 & -3.113 & .002 \\
\hline \multirow[t]{2}{*}{ AZ0706 } & 10 & 2007 & $\beta$ & .006 & .003 & 1.67 & .097 \\
\hline & & & Y & .005 & .005 & .936 & .351 \\
\hline \multirow[t]{2}{*}{ AZ0801 } & 11 & 2008 & $\beta$ & .030 & .006 & 4.417 & $<.0001$ \\
\hline & & & $\gamma$ & -.036 & .008 & -4.618 & $<.0001$ \\
\hline \multirow[t]{2}{*}{ AZ0810 } & 12 & 2008 & $\beta$ & .042 & .006 & 6.841 & $<.0001$ \\
\hline & & & Y & .075 & .012 & 6.467 & $<.0001$ \\
\hline \multirow[t]{2}{*}{ AZ0811 } & 13 & 2008 & $\beta$ & .008 & .003 & 2.318 & .021 \\
\hline & & & Y & .024 & .004 & 5.741 & $<.0001$ \\
\hline \multirow[t]{2}{*}{ AZ0816 } & 14 & 2008 & $\beta$ & .029 & .004 & 6.781 & $<.0001$ \\
\hline & & & $\gamma$ & .068 & .006 & 11.412 & $<.0001$ \\
\hline \multirow[t]{2}{*}{ AZ0809 } & 15 & 2008 & $\beta$ & .017 & .006 & 2.796 & $<.007$ \\
\hline & & & $\gamma$ & .009 & .008 & 1.224 & .226 \\
\hline \multirow[t]{2}{*}{ AZ0802 } & 16 & 2008 & $\beta$ & .005 & .001 & 2.714 & .007 \\
\hline & & & Y & .011 & .002 & 4.377 & $<.0001$ \\
\hline \multirow[t]{2}{*}{ AZ0812 } & 17 & 2008 & $\beta$ & .009 & .003 & 2.699 & .007 \\
\hline & & & $\gamma$ & .029 & .006 & 5.557 & $<.0001$ \\
\hline \multirow[t]{2}{*}{ AZ0813 } & 18 & 2008 & $\beta$ & .017 & .005 & 3.095 & .002 \\
\hline & & & Y & .028 & .01 & 3.110 & .002 \\
\hline \multirow[t]{2}{*}{ NM0810 } & 19 & 2008 & $\beta$ & .006 & .004 & 1.518 & .131 \\
\hline & & & $\gamma$ & .033 & .008 & 3.812 & .0001 \\
\hline \multirow[t]{2}{*}{ AZ0903 } & 20 & 2009 & $\beta$ & .020 & .012 & 1.638 & .105 \\
\hline & & & $\gamma$ & .001 & .020 & .089 & .929 \\
\hline
\end{tabular}


Table 1 The mode and strength of selection on trophic morphology in natural ponds (Continued)

\begin{tabular}{lllllllr}
\hline AZ0902 & 21 & 2009 & $\beta$ & .017 & .002 & 6.017 & $<.0001$ \\
& & $\gamma$ & .016 & .006 & 2.692 & .007 \\
AZ0904 & 22 & 2009 & $\beta$ & .012 & .002 & 5.326 & $<.0001$ \\
& & & $\gamma$ & -.010 & .002 & -4.917 & $<.0001$
\end{tabular}

The pond name, map ID corresponding to Figure 1, and year of collection are given for each population, along with the regression terms ( $\beta / \gamma$ ), estimated selection differential for each term, its standard error (SE), $t$-statistic and probability of rejecting the null hypothesis that the estimated differential is zero. For quadratic regressions, positive selection differentials signify disruptive selection and the quadratic regression coefficient is doubled to calculate the quadratic selection differential $(\gamma)$ and the associated standard error $(\mathrm{SE})$ is also doubled. Bolding signifies statistical significance.

We sought to determine the prevalence of disruptive selection among natural populations of spadefoot toads. We also sought to establish whether the strength of disruptive selection was positively associated with the intensity of intraspecific competition within individual populations.

Disruptive selection favoring extreme resource-use phenotypes, as measured here by using larval body size to estimate tadpole fitness (see Methods), was widespread in the spadefoot toad populations surveyed in this study. Indeed, we documented disruptive selection on tadpole trophic morphology in 11 of 15 populations surveyed (Figure 1). These results, together with those of previous studies $[18,21,46]$, strongly support the role of disruptive selection in the evolution of resource polymorphism in Spea tadpoles [46], and add to the evidence that disruptive selection can be widespread within certain systems [22].

Although disruptive selection was prevalent in our surveyed populations, we also found considerable variation

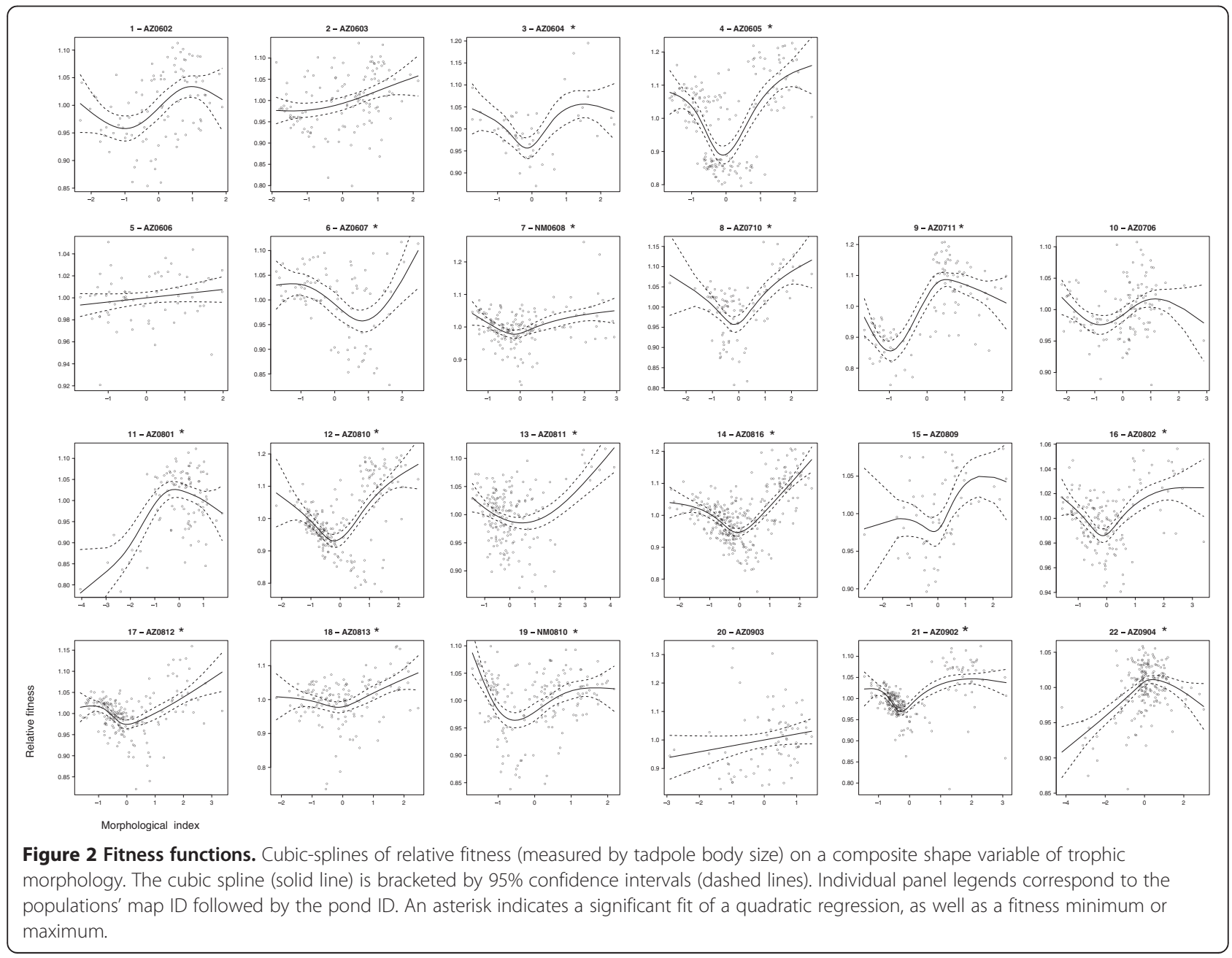



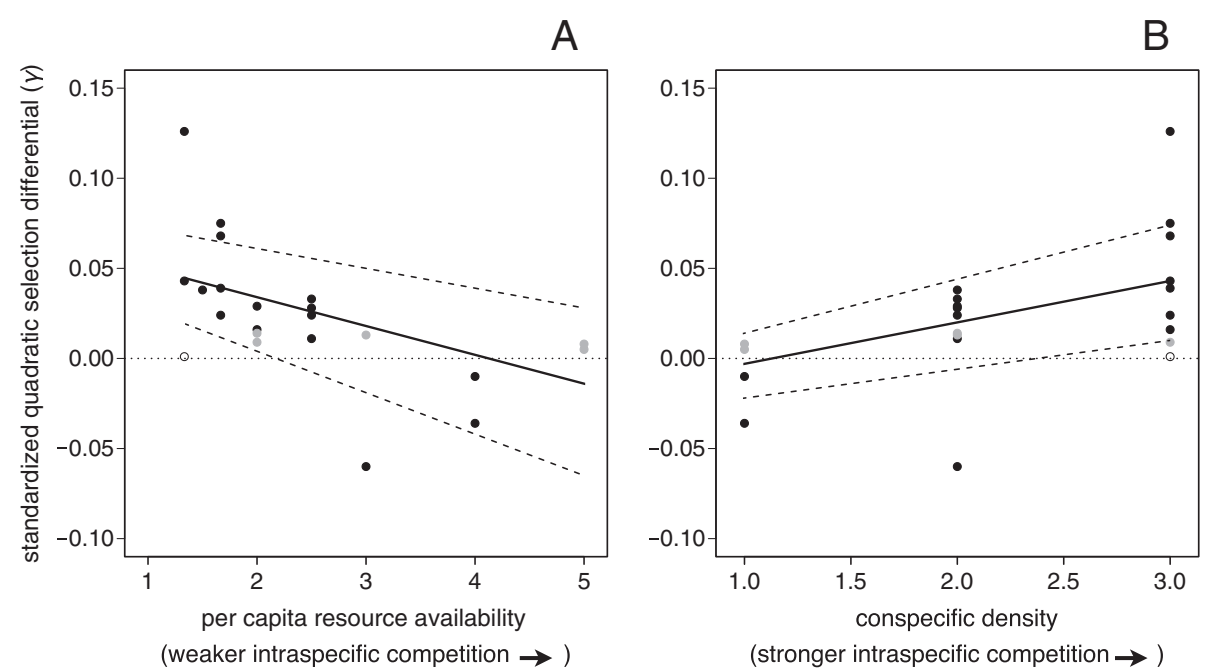

Figure 3 Intensity of disruptive selection as function of the intensity of competition. The relationship between quadratic selection and two measures of intraspecific competition. The intensity of disruptive selection on trophic morphology was greater in ponds with (A) lower per capita resource density and (B) higher conspecific density. Standardized quadratic selection differentials above the dotted line are positive and indicate disruptive selection, while those below the line are negative and indicate stabilizing selection. Significant selection differentials (black), nonsignificant selection differentials (grey) and significant selection differentials with no fitness minimum or maximum in the range of the data (open) are all shown. Solid and dashed lines show respectively, the median and 95\% confidence intervals of the regression coefficient obtained from bootstrapping. Each point in the analysis was weighted by the populations' sample size.

in both the mode and magnitude of quadratic selection (Figures 1, 2; Table 1). This variation correlated predictably with the intensity of intraspecific competition within individual populations. Specifically, disruptive selection was strongest in ponds with the highest conspecific density and lowest per capita resource density (Figure 3A, B). In contrast, stabilizing selection favoring intermediate resource-use phenotypes was found in ponds where intraspecific competition was weak (Figure 3A, B). These results therefore confirm theory suggesting that intraspecific competition can drive disruptive selection (see Introduction). Furthermore, our results suggest that disruptive selection is likely to occur in habitats relatively free of predators, heterospecific competitors, or other ecological factors that depress population size and that therefore potentially weaken intraspecific competition.

Interestingly, the two extreme ecomorphs do not appear to be equally favored by disruptive selection. Previous studies found that carnivores achieve greater survival and are generally (although not always) larger at metamorphosis than either intermediates or omnivores $[18,21]$. We similarly found that a directional component to selection favoring carnivores may be widespread (Figure 2; Table 1). Yet, if one extreme morph (the carnivore morph) has higher fitness than the other extreme morph (the omnivore morph), how is resource polymorphism maintained in this system?

Several factors examined in previous studies may help maintain both omnivores and carnivores within the same population. First, as carnivores become increasingly common in any given pond, and as competition among carnivores for the limited shrimp resource thereby becomes more intense, negative frequencydependent selection favors omnivores [43]. In support of these earlier findings, we found evidence in this study for pure disruptive selection (with no directional component) in two ponds, and disruptive selection with a directional component favoring omnivores in another (Figure 2; Table 1). Moreover, although carnivores benefit by gaining access to the more profitable shrimp resource (which could explain why they often achieve larger body size), additional experiments have shown that carnivores also experience greater competition with other carnivores than omnivores do with other omnivores [37]. In this way, negative frequency-dependent selection maintains both morphs within the same population [43]. Second, fitness trade-offs are associated with each of these phenotypic alternatives. Compared to carnivores, omnivores invest more into abdominal fat bodies, which increases post-metamorphic resistance to starvation [43]. Thus, although carnivores may often have higher survival before metamorphosis (the life stage at which we estimated selection in the present study), omnivores may have higher survival immediately after metamorphosis, possibly balancing their lower premetamorphic survival [43]. Generally, frequencydependent selection, coupled with fitness trade-offs, likely contribute to the evolutionary maintenance of many resource polymorphisms [4]. 
A deeper problem requiring explanation, however, is why intermediate phenotypes persist in the face of widespread disruptive selection against them (Figure 1). Although morph determination is environmentally triggered-carnivore development is induced when a tadpole consumes fairy shrimp [50]—considerable heritable variation in the response to this cue exists in natural populations $[48,51]$, suggesting that the propensity to produce intermediate phenotypes could, in principle, be eliminated by natural selection. A possible resolution to this problem is that sexual selection may lead to random mating in regard to larval phenotype, recreating tadpoles likely to develop as intermediate phenotypes in each generation. Specifically, in our populations, female $S$. multiplicata prefer to mate with males in good condition, irrespective of the females' own condition [54,55]. Therefore, female $S$. multiplicata will tend to mate with males of good condition, potentially regardless of either the male's larval phenotype or the female's own larval phenotype (e.g., a good-condition adult male might have been either an omnivore or a carnivore as a tadpole). This directional selection on male condition (exerted by female preferences for mates) might thereby oppose disruptive selection acting on the larvae. Indeed, directional sexual selection may often oppose disruptive natural selection in many populations [56], which may explain why an increasing number of cases have been reported in which assortative mating by resource-use phenotype was expected but not found $[57,58]$.

Spatial and temporal variation in the mode of selection may also help explain the persistence of intermediate larval phenotypes. For example, stabilizing selection favoring individuals with intermediate phenotypes was detected in some ponds (Figure 1, Figure 2), specifically those lacking strong intraspecific competition (Figure 3A, 3B). Stabilizing selection was also detected in ponds in which $S$. multiplicata co-occurs with a congener, Spea bombifrons [21]. Such mixed-species ponds may be within a few kilometers of pure $S$. multiplicata ponds in the San Simon Valley of southeastern Arizona [53]. Thus, S. bombifrons migrants may occasionally colonize nearby pure $S$. multiplicata ponds, thereby changing the selective regime within the pond to one in which intermediate phenotypes are favored. Indeed, $S$. bombifrons occurred along with $S$. multiplicata within some of the ponds sampled for this study as recently as 30 years ago [59]. Thus, because the mode and direction of selection is spatially and temporally variable, intermediate phenotypes may persist in the population.

Finally, limits in the ability of tadpoles to respond appropriately through phenotypic plasticity to variation in both fairy shrimp density [50] and their competitive environment (i.e., conspecific density and morph frequency) may explain the persistence of intermediate phenotypes in natural ponds. For instance, spatial and temporal variation in the density of fairy shrimp within a pond could give some individuals a head start in carnivore expression early in development. Once tadpoles increase their mobility as they grow and develop, tadpoles encountering fairy shrimp later may begin to develop as carnivores, but these late-developing carnivores might end up being outcompeted by earlier-developing carnivores. If these individuals are deprived of fairy shrimp, their carnivore-like features begin to regress, and they assume an intermediate phenotype [43]. Generally, intermediate phenotypes may be difficult to eradicate via disruptive selection when limits exist in the ability of individuals to assess and respond adaptively to their competitive environment though phenotypic plasticity. Further studies are needed to test these ideas.

\section{Conclusions}

Although disruptive selection has long been viewed as a potential generator of biological diversity, it has received relatively little attention, and, consequently, little is known regarding its prevalence or causes within specific systems. Using spadefoot toad tadpoles as a model system, we found that disruptive selection can be prevalent in the wild, and that its occurrence is predictably associated with a specific ecological factor: strong intraspecific competition. Given that intraspecific competition is common in many natural populations-and frequently strong [60,61] - our results therefore imply that disruptive selection may be a more important force contributing to the origin and maintenance of biological diversity than is currently appreciated.

\section{Methods}

All procedures were carried out in compliance with the Institutional Animal Care and Use Committee at the University of North Carolina under application 06047.0-A. Field collections were conducted under New Mexico collecting permit 1857 and Arizona collecting permit SP604848.

\section{Field surveys}

We collected $S$. multiplicata tadpoles during summers 2006-2009 from 15 ephemeral ponds in the San Simon Valley (Figure 1). In all 15 ponds, S. multiplicata was the only species of Spea present. Six ponds were sampled across multiple years for a total of 22 collections (Figure 1; Table 2). We sampled each pond on a single day 16-20 days after breeding had occurred (Spea breed only a single time within a pond each season, shortly after the pond fills with rainwater). Within each pond, we sampled tadpoles from five randomly selected sites throughout the pond using a hand-held dip net (median sample size $=130$ tadpoles). We sacrificed the tadpoles 
Table 2 Summary of tadpole collections and ecological parameters in natural ponds

\begin{tabular}{|c|c|c|c|c|c|c|}
\hline Pond & Map ID & Year & $\mathbf{N}$ & Tadpole density & Fairy shrimp density & Cover (\%) \\
\hline AZ0602 & 1 & 2006 & 93 & 2 & 3 & .8 \\
\hline AZ0603 & 2 & 2006 & 124 & 1 & 2 & .75 \\
\hline AZ0604 & 3 & 2006 & 50 & 2 & 3 & .5 \\
\hline AZ0605 & 4 & 2006 & 176 & 3 & 2 & .6 \\
\hline AZ0606 & 5 & 2006 & 94 & 2 & 1 & .9 \\
\hline AZ0607 & 6 & 2006 & 102 & 2 & 1 & .4 \\
\hline NM0608 & 7 & 2006 & 165 & 3 & 2 & 1 \\
\hline AZO710 & 8 & 2007 & 78 & 3 & 1 & .9 \\
\hline AZ0711 & 9 & 2007 & 99 & 2 & 3 & 1 \\
\hline AZ0706 & 10 & 2007 & 125 & 1 & 2 & .7 \\
\hline AZ0801 & 11 & 2008 & 99 & 1 & 2 & 1 \\
\hline AZ0810 & 12 & 2008 & 213 & 3 & 2 & .8 \\
\hline AZ0811 & 13 & 2008 & 181 & 3 & 3 & .5 \\
\hline AZ0816 & 14 & 2008 & 297 & 3 & 2 & 1 \\
\hline AZ0809 & 15 & 2008 & 59 & 3 & 3 & 1 \\
\hline AZ0802 & 16 & 2008 & 150 & 2 & 2 & .7 \\
\hline AZ0812 & 17 & 2008 & 188 & 2 & 2 & .6 \\
\hline AZ0813 & 18 & 2008 & 135 & 2 & 2 & 1 \\
\hline NM0810 & 19 & 2008 & 169 & 2 & 2 & .8 \\
\hline AZ0903 & 20 & 2009 & 78 & 3 & 1 & .75 \\
\hline AZ0902 & 21 & 2009 & 211 & 3 & 3 & 1 \\
\hline AZ0904 & 22 & 2009 & 192 & 1 & 1 & 1 \\
\hline
\end{tabular}

For each population the pond name, map ID corresponding to Figure 1, sampling year, tadpole sample size (N), tadpole density, fairy shrimp density, and percentage of vegetative cover (Cover) are shown. We assigned numerical values to our estimates of tadpole and shrimp abundance such that "high" $=3$, "medium" = 2 and "low" = 1.

immediately after collection by immersion in a $0.1 \%$ aqueous solution of tricane methanesulfonate (MS 222) and preserved them in $95 \%$ ethanol. We used this random sampling technique to estimate the density of $S$. multiplicata tadpoles in each pond as 'high', 'moderate' and 'low' [46]. Additionally, we determined the range of available resources in each pond by estimating abundances of fairy shrimp and detritus, which are the two main resources on which Spea tadpoles feed. We estimated fairy shrimp abundance by sweeping a net throughout each pond and categorizing shrimp densities as "high," "moderate," and, "low". These subjective estimates were corroborated by previously published intensive, quantitative sampling [50,53]. We assessed the availability of detritus by estimating the percent vegetative cover in a twenty meter radius around each pond's circumference and categorized each pond as having either "high" (67\%-100\% cover), "moderate" (34\%-66\% cover) or, "low" (0\%-33\% cover) detritus (ponds with more vegetation tend to have more detritus; see [53]).

We calculated an estimate of per capita resource abundance for each pond by dividing the sum of fairy shrimp and detritus abundance ("high" $=3$, "medium" $=2$ and "low" =1) by S. multiplicata tadpole density ("high" = 3, "medium" = 2 and "low" $=1$ ). We subsequently used this score to test our prediction that ponds with high levels of intraspecific competition experienced the most intense disruptive selection.

\section{Evaluating the prevalence of disruptive selection}

To evaluate the prevalence of disruptive selection on trophic morphology across ponds, we first calculated a composite index of trophic morphology, separately for each pond, following previously described methods $[18,21,46]$. Briefly, we began by measuring each tadpole's snout-vent-length (SVL) using hand-held digital calipers. For each tadpole, we additionally measured the width of the orbitohyoideus $(\mathrm{OH})$ muscle and characterized the shape of each tadpole's keratinized mouthparts (MP) on an ordinal scale from one (most omnivore-like) to five (most carnivore-like), and counted the number of denticle rows (DR) surrounding the keratinized mouthparts. We standardized $\mathrm{OH}$ for body size (SVL) by regressing $\ln$ (i.e., natural $\log$ ) $\mathrm{OH}$ on $\ln \mathrm{SVL}$ and used the resulting residuals for the subsequent analyses (these residuals were distributed normally). We then combined the MP 
Table 3 Principal component analysis of trophic morphology

\begin{tabular}{|c|c|c|c|c|c|c|}
\hline Pond & Map ID & PC & MP eigenvector loading & residual $\mathrm{OH}$ eigenvector loading & DR eigenvector loading & $\%$ variance explained \\
\hline \multirow[t]{3}{*}{$\overline{A Z 0602}$} & 1 & 1 & .675 & .692 & -.252 & 49.5 \\
\hline & & 2 & -.252 & -.104 & -.961 & 32.4 \\
\hline & & 3 & -.692 & .713 & .104 & 18.0 \\
\hline \multirow[t]{3}{*}{ AZ0603 } & 2 & 1 & .707 & .707 & - & 82.0 \\
\hline & & 2 & .707 & -.707 & - & 17.9 \\
\hline & & 3 & - & - & - & - \\
\hline \multirow[t]{3}{*}{ AZ0604 } & 3 & 1 & .594 & .647 & -.477 & 62.5 \\
\hline & & 2 & -.511 & -.154 & -.845 & 25.7 \\
\hline & & 3 & .620 & -.746 & -.238 & 11.6 \\
\hline \multirow[t]{3}{*}{ AZ0605 } & 4 & 1 & .576 & .644 & -.502 & 59.9 \\
\hline & & 2 & -.581 & -.108 & -.806 & 25.9 \\
\hline & & 3 & .574 & -.756 & -.312 & 14.2 \\
\hline \multirow[t]{3}{*}{ AZ0606 } & 5 & 1 & .707 & .707 & - & 76.5 \\
\hline & & 2 & .707 & -.707 & - & 23.5 \\
\hline & & 3 & - & - & - & - \\
\hline \multirow[t]{3}{*}{ AZ0607 } & 6 & 1 & .691 & -.466 & -.372 & 52.2 \\
\hline & & 2 & -.057 & -.057 & -.882 & 31.7 \\
\hline & & 3 & -.720 & -.882 & -.287 & 15.7 \\
\hline \multirow[t]{3}{*}{ NM0608 } & 7 & 1 & .701 & .690 & -.176 & 52.3 \\
\hline & & 2 & .058 & -.190 & -.979 & 32.8 \\
\hline & & 3 & .710 & 697 & -.093 & 14.7 \\
\hline \multirow[t]{3}{*}{ AZ0710 } & 8 & 1 & .600 & .591 & -.538 & 64.0 \\
\hline & & 2 & -.318 & -.441 & -.838 & 20.8 \\
\hline & & 3 & -.733 & .675 & -.076 & 15.14 \\
\hline \multirow[t]{3}{*}{ AZ0711 } & 9 & 1 & .590 & .475 & -.652 & 64.3 \\
\hline & & 2 & .530 & -.837 & -.130 & 25.7 \\
\hline & & 3 & -.608 & -.269 & -.746 & 9.8 \\
\hline \multirow[t]{3}{*}{ AZ0706 } & 10 & 1 & .560 & .608 & -.561 & 71.0 \\
\hline & & 2 & .712 & -.007 & .701 & 17.8 \\
\hline & & 3 & -.423 & .793 & .438 & 11.1 \\
\hline \multirow[t]{3}{*}{ AZ0801 } & 11 & 1 & .534 & .589 & -.605 & 44.8 \\
\hline & & 2 & .836 & -.470 & .279 & 28.7 \\
\hline & & 3 & -.120 & -.656 & -.745 & 26.3 \\
\hline \multirow[t]{3}{*}{ AZ0810 } & 12 & 1 & .588 & .547 & -.594 & 68.0 \\
\hline & & 2 & .442 & -.833 & -.330 & 18.4 \\
\hline & & 3 & .676 & .068 & .732 & 13.4 \\
\hline \multirow[t]{3}{*}{ AZ0811 } & 13 & 1 & .597 & .590 & -.542 & 53.5 \\
\hline & & 2 & -.327 & -.437 & -.837 & 24.9 \\
\hline & & 3 & .731 & -.678 & .068 & 21.5 \\
\hline \multirow[t]{3}{*}{ AZ0816 } & 14 & 1 & .597 & .551 & -.581 & 66.3 \\
\hline & & 2 & .242 & -.815 & -.525 & 19.0 \\
\hline & & 3 & -.764 & .172 & -.621 & 14.5 \\
\hline \multirow[t]{2}{*}{ AZ0809 } & 15 & 1 & .593 & .558 & -.578 & 49.4 \\
\hline & & 2 & -.211 & .802 & .557 & 26.1 \\
\hline
\end{tabular}


Table 3 Principal component analysis of trophic morphology (Continued)

\begin{tabular}{|c|c|c|c|c|c|c|}
\hline & & 3 & -.776 & .208 & -.595 & 24.4 \\
\hline \multirow[t]{3}{*}{ AZ0802 } & 16 & 1 & .628 & .684 & -.369 & 55.9 \\
\hline & & 2 & -.429 & -.090 & -.898 & 30.9 \\
\hline & & 3 & -.648 & .723 & .237 & 13.1 \\
\hline \multirow[t]{3}{*}{ AZ0812 } & 17 & 1 & .641 & .501 & -.580 & 59.9 \\
\hline & & 2 & -.125 & .815 & .565 & 25.6 \\
\hline & & 3 & .756 & -.290 & .585 & 14.4 \\
\hline \multirow[t]{3}{*}{ AZ0813 } & 18 & 1 & .550 & .777 & -.304 & 38.7 \\
\hline & & 2 & -.607 & .122 & -.784 & 36.6 \\
\hline & & 3 & -.572 & .617 & .539 & 24.6 \\
\hline \multirow[t]{3}{*}{ NM0810 } & 19 & 1 & .577 & .595 & -.558 & 76.0 \\
\hline & & 2 & -.571 & -.192 & -.797 & 14.3 \\
\hline & & 3 & .582 & -.779 & -.229 & 9.6 \\
\hline \multirow[t]{3}{*}{ AZ0903 } & 20 & 1 & .404 & .710 & -.576 & 47.5 \\
\hline & & 2 & .814 & .006 & .579 & 33.5 \\
\hline & & 3 & -.415 & .703 & .576 & 18.6 \\
\hline \multirow[t]{3}{*}{ AZ0902 } & 21 & 1 & .591 & .556 & -.583 & 76.5 \\
\hline & & 2 & .307 & -.824 & -.475 & 14.0 \\
\hline & & 3 & .745 & -.101 & .658 & 9.4 \\
\hline \multirow[t]{3}{*}{ AZ0904 } & 22 & 1 & .557 & .588 & -.585 & 49.2 \\
\hline & & 2 & .828 & -.350 & .436 & 26.1 \\
\hline & & 3 & -.051 & .728 & .683 & 24.5 \\
\hline
\end{tabular}

For each population the pond name, map ID corresponding to Figure 1, principal component axis (PC), trait loadings and \% variance explained are shown.

scores, DR counts, and residuals of $\ln \mathrm{OH}$ regressed on ln SVL, into a single multivariate shape variable (the "morphological index"; see [18]) with a principal component analysis using the correlation matrix, and standardized to have a standard deviation of one. Our morphological index for each pond was the first principal component. Larger values of PC1 correspond to more carnivore-like tadpoles, with larger $\mathrm{OH}$ muscles (corrected for body size), fewer denticle rows, and more serrated, notched mouthparts. In contrast smaller values of PC1 correspond to more omnivore-like tadpoles, with smaller $\mathrm{OH}$ muscles (corrected for body size), more denticle rows and smooth mouthparts (Table 3).

We were unable to obtain denticle row counts for tadpoles from two collections (AZ0603 and AZ0606, see Table 3). Therefore, for these two collections we calculated an alternative morphological index using only MP scores and residual values of $\ln \mathrm{OH}$ corrected for $\ln$ SVL [21]. We calculated a single multivariate shape variable by combining the MP scores and residuals $\mathrm{OH}$ values by again using a principal component analysis as described above. As before, the morphological index for each of the two ponds was the first principal component. Larger values of $\mathrm{PC} 1$ correspond to more carnivore-like tadpoles, with larger $\mathrm{OH}$ muscles (corrected for body size), and more serrated, notched mouthparts. In contrast smaller values of PC1 correspond to more omnivore-like tadpoles, with smaller $\mathrm{OH}$ muscles (corrected for body size), and smooth mouthparts (Table 3).

We estimated the mode and magnitude of selection acting on tadpole trophic morphology in each pond using body size as a fitness proxy [ln SVL; see 21, 62]. Body size is positively correlated with fitness in many species [62], including in Spea. For example, relative to tadpoles within the same population that are larger, tadpoles that are smaller in body size have a lower probability of survival, both before [63] and after metamorphosis [64]. Moreover, smaller tadpoles tend to be less developmentally advanced, and there is a premium on rapid development in the ephemeral ponds in which Spea multiplicata typically breed [43]. Additionally, in Spea, adult size is positively correlated with mating success in males [54] and fecundity in females [63]. Finally, a previous field experiment, in which tadpoles of different morphotypes were marked and recaptured within a natural pond, established that the relationship between morphology and body size mirrored that between body size and survival [18]. In short, body size is a reliable proxy for fitness in this system. 


\begin{tabular}{|c|c|c|c|c|c|c|}
\hline Pond & Map ID & $F_{\min }$ & $P_{\min }$ & $F_{\max }$ & $P_{\max }$ & $\begin{array}{l}\text { fitness } \\
\mathrm{min} / \mathrm{max}\end{array}$ \\
\hline AZ0604 & 3 & 5.124 & .028 & 10.522 & .002 & $Y$ \\
\hline AZ0605 & 4 & 48.257 & $<.0001$ & 80.282 & $<.0001$ & Y \\
\hline AZ0607 & 6 & 12.177 & .0007 & 5.311 & .024 & Y \\
\hline NM0608 & 7 & 7.810 & .005 & 16.573 & $<.0001$ & Y \\
\hline AZ0710 & 8 & 12.492 & .0006 & 30.103 & $<.0001$ & Y \\
\hline AZ0711 & 9 & 28.468 & $<.0001$ & 1.559 & .214 & $N$ \\
\hline AZ0801 & 11 & 31.686 & $<.0001$ & 8.232 & .005 & Y \\
\hline AZ0810 & 12 & 23.971 & $<.0001$ & 61.126 & $<.0001$ & Y \\
\hline AZ0811 & 13 & 21.384 & $<.0001$ & 38.224 & $<.0001$ & Y \\
\hline AZ0816 & 14 & 85.242 & $<.0001$ & 172.109 & $<.0001$ & Y \\
\hline AZ0802 & 16 & 24.155 & $<.0001$ & 10.773 & .001 & Y \\
\hline AZ0812 & 17 & 20.838 & $<.0001$ & 36.537 & $<.0001$ & Y \\
\hline AZ0813 & 18 & 5.154 & .024 & 14.562 & .0002 & Y \\
\hline NM0810 & 19 & 10.912 & .001 & 16.500 & $<.0001$ & Y \\
\hline AZ0903 & 20 & .083 & .773 & .317 & .574 & $N$ \\
\hline AZ0902 & 21 & 1.691 & .194 & 14.560 & .0001 & $\mathrm{~N}$ \\
\hline AZ0904 & 22 & 39.010 & $<.0001$ & 9.252 & .002 & Y \\
\hline
\end{tabular}

For each population the pond name, map ID corresponding to Figure 1, test statistics and results evaluating if the null hypotheses that fitness minimum/ maximum lie at extreme minimum and maximum phenotypic values (rather than within the observed range of the data) can be rejected.

To estimate the magnitude and mode of selection, we ran separate linear and quadratic regression (including both linear and quadratic terms for the latter) of relative fitness (ln SVL/mean ln SVL) onto the morphological index for each pond $[65,66]$. We obtained standardized selection differentials for linear and quadratic selection from these regressions of relative fitness on trophic morphology. We doubled the quadratic regression coefficients to obtain quadratic selection differentials $(\gamma)$ $[66,67]$.

A significant, standardized linear selection differential (ß) indicates that directional selection is acting on trophic morphology [66]. Selection on trophic morphology might be disruptive when the quadratic selection differential $(\gamma)$ is significantly positive, and stabilizing when it is negative [66]. However, a significant $\gamma$ is necessary, but not sufficient to indicate the presence of quadratic selection where intermediate phenotypes are at the fitness maximum/minimum [68]. Therefore, for each pond, we fit cubic splines between the morphological index and fitness along with 95\% confidence intervals to visualize the selective surface [69]. Cubic spline analysis is less sensitive to outliers and allows estimation of a fitness function without an a priori assumption about the function's shape [69]. We visually inspected these plots to determine if there was an intermediate fitness minimum or maximum within the range of the data (Figure 2). For the ponds with significant quadratic regression differentials we also applied a constrained regression method to statistically evaluate if the null hypotheses that fitness minimum/maximum lie at extreme phenotypic values (rather than within the observed range of the data) can be rejected (Table 4) $[68,70]$. Where the graphical and statistical methods conflicted (in two cases) we favored our visual evaluation of the cubic splines because the constrained regression method is sensitive to deviations from the assumptions of parametric statistical tests that do not affect the nonparametric cubic spline approach [69].

\section{Evaluating whether competition predicts the strength of disruptive selection}

Finally, we sought to test our prediction regarding the ecological correlates of disruptive selection. Specifically, we predicted that disruptive selection would be strongest in ponds with intense intraspecific competition. To test this prediction, we fit separate linear regressions of the quadratic selection differentials onto two estimates of intraspecific competition: (1) conspecific density, and (2) per capita resource density. Our underlying assumption was that intraspecific competition would be more intense the higher the density of conspecifics and the lower the per capita resource density within a pond.

We weighted the selection differentials in each regression described above by the square root of our sample size for each pond. We did so because confidence in the estimation of both the sign and magnitude of selection differentials is lower for those ponds with small sample sizes then for those with larger sample sizes.

To further evaluate our models we present confidence intervals obtained from bootstrapping for each analysis to account for potential pseudo-replication introduced by using temporal replicates from the same pond. For the regression models used in testing our two predictions, we sampled a single selection differential from each of the fifteen unique ponds, fit the regression, and extracted the regression coefficients. We resampled the selection differentials and refit each model 1000 times and then estimated 95\% confidence intervals for each regression coefficient. We assessed significance by asking if the range of the confidence interval excluded zero. All statistical analyses were performed using $\mathrm{R}$ (version 2.15.0) [71].

\section{Competing interests}

The authors declare that they have no competing interests.

\section{Authors' contributions}

RM and DP designed the study, did field work, and wrote the paper. RM analyzed the data. Both authors approved the final version of the manuscript. 


\section{Acknowledgements}

We thank S. Diamond and K. Pfennig for helpful comments and K. Pfennig, A. Chunco, J. Paull, S. Suni, S. Brown, and the staff and volunteers of the Southwestern Research Station for field assistance. We also thank the Game and Fish Departments of Arizona and New Mexico for providing scientific collecting permits and the National Science Foundation (to DWP), the SWRS Student Support Fund, UNC Smith Graduate Research Grant, and the UNC Biology department H.V. Wilson Award (to RAM) for funding.

Received: 19 March 2012 Accepted: 19 July 2012

Published: 2 August 2012

\section{References}

1. Mather K: Polymorphism as an outcome of disruptive selection. Evolution 1955, 9(1):52-61.

2. Rueffler C, Van Dooren TJM, Leimar O, Abrams PA: Disruptive selection and then what? Trends in Ecology and Evolution 2006, 21:238-245.

3. Bürger R, Gimelfarb A: The effects of intraspecific competition and stabilizing selection on a polygenic trait. Genetics 2004, 167:1425-1443.

4. Smith TB, Skúlason S: Evolutionary significance of resource polymorphisms in fishes, amphibians, and birds. Annu Rev Ecol Syst 1996 27:111-133.

5. Thoday JM: Effects of disruptive selection: experimental production of a polymorphic population. Nature 1958, 181:1124-1125.

6. Cooper IA, Gilman RT, Boughman JW: Sexual dimorphism and speciation on two ecological coins: patterns from nature and theoretical predictions. Evolution 2011, 65:2553-2571.

7. Van Valen LM: Morphological variation and width of ecological niche. Am Nat 1965, 99:377-390

8. Bolnick DI, Doebeli M: Sexual dimorphism and adaptive speciation: two sides of the same ecological coin. Evolution 2003, 57:2433-2449.

9. Dieckmann U, Doebeli M: On the origin of species by sympatric speciation. Nature 1999, 400:354-357.

10. Rosenzweig ML: Competitive speciation. Biol I Linn Soc 1978, 10:274-289.

11. Maynard Smith J: Disruptive selection, polymorphism and sympatric speciation. Nature 1962, 195:60-62.

12. Udovic D: Frequency-dependent selection, disruptive selection, and the evolution of reproductive isolation. Am Nat 1980, 116:621-641.

13. Kingsolver JG, Diamond SE: Phenotypic selection in natural populations: what limits directional selection? Am Nat 2011, 177:346-357.

14. Kingsolver JG, Hoekstra HE, Hoekstra JM, Berrigan D, Vignieri N, Hill CE, Hoang A, Gilbert P, Beerli P: The strength of phenotypic selection in natural populations. Am Nat 2001, 157:245-261.

15. Calsbeek R, Smith TB: Experimentally replicated disruptive selection on performance traits in a Caribbean lizard. Evolution 2008, 62:478-484.

16. Cucherousset J, Acou A, Blachet S, Britton JA, Beaumont WRC, Gozlan RE: Fitness consequences of individual specialization in resource use and trophic morphology in European eels. Oecologia 2011, 167:75-84.

17. Hendry AP, Huber SK, De Leon LF, Herrel A, Podos J: Disruptive selection in a bimodal population of Darwin's finches. Proceedings of The Royal Society, Series B 2009, 276:753-759.

18. Martin RA, Pfennig DW: Disruptive selection in natural populations: the roles of ecological specialization and resource competition. Am Nat 2009, 174:268-281.

19. Medel R, Botto-Mahan C, Kalin-Arroyo M: Pollinator-mediated selection on the nectar guide phenotype in the Andean monkey flower, Mimulusluteus. Ecology 2003, 84:1721-1732.

20. Smith TB: Disruptive selection and the genetic basis of bill size polymorphism in the African finch Pyrenestes. Nature 1993, 363:618-620.

21. Pfennig DW, Rice AM, Martin RA: Field and experimental evidence for competition's role in phenotypic divergence. Evolution 2007, 61:257-271.

22. Bolnick DI, Lau OL: Predictable patterns of disruptive selection in stickleback in postglacial lakes. Am Nat 2008, 172:1-11.

23. Svanbäck R, Persson L: Population density fluctuations change the selection gradient in Eurasian perch. Am Nat 2009, 173:507-516.

24. Duffy MA, Brassil CE, Hall SR, Tessier AJ, Caceres CE, Conner JK: Parasitemediated disruptive selection in a natural Daphnia population. BMC Evol Biol 2008, 8:80

25. Doebeli M, Dieckmann U: Evolutionary branching and sympatric speciation caused by different types of ecological interactions. Am Nat 2000, 156(Suppl):77-101.
26. Wilson DS, Turelli M: Stable underdominance and the evolutionary invasion of empty niches. Am Nat 1986, 127:835-850.

27. Abrams PA, Matsuda $H$, Harada Y: Evolutionarily unstable fitness maxima and stable fitness minima of continuous traits. Evol Ecol 1993, 7:465-487.

28. Antonovics J, Thrall PH: Cost of resistance and the maintenance of genetic polymorphism in host-pathogen systems. Proc R Soc Lond Ser BBiol Sci 1994, 257:105-110.

29. Abrams PA, Rueffler C, Kim G: Determinants of the strength of disruptive and/or divergent selection arising from resource competition. Evolution 2008, 62:1571-1586.

30. Ackermann M, Doebeli M: Evolution of niche width and adaptive diversification. Evolution 2004, 58:2599-2612.

31. Benkman CW: Are the ratios of bill crossing morphs in crossbills the result of frequency-dependent selection? Evol Ecol 1996, 10:119-126.

32. Hulsey CD, Hendrickson DA, de Leon FJG: Trophic morphology, feeding performance and prey use in the polymorphic fish Herichthys minckleyi. Evol Ecol Res 2005, 7:303-324.

33. Robinson BW, Wilson DS, Shea GO: Trade-offs of ecological specialization: an intraspecific comparison of pumpkinseed sunfish phenotypes. Ecology 1996, 77:170-178.

34. Calsbeek R: Experimental evidence that competition and habitat use shape the individual fitness surface. J Evol Biol 2009, 22:97-108.

35. Bolnick DI: Can intraspecific competition drive disruptive selection? An experimental test in natural populations of sticklebacks. Evolution 2004, 58:608-618.

36. Levins R: Evolution in changing environments. Princeton, N.J.: Princeton University Press; 1968.

37. Paull JS, Martin RA, Pfennig DW: Increased competition as a cost of specialization during the evolution of resource polymorphism. Biol I Linn Soc, in press.

38. Day T, Young KA: Competitive and facilitative evolutionary diversification. BioScience 2004, 54:101-109.

39. Doebeli M: Adaptive diversification. Princeton, NJ: Princeton University Press; 2011.

40. Elmer KR, Lehtonen TK, Kautt AF, Harrod C, Meyer A: Rapid sympatric ecological differentiation of crater lake cichlid fishes within historic times. BMC Biology 2010, 8:60

41. Hori M: Frequency-dependent natural selection in the handedness of scale-eating cichlid fish. Science 1993, 260:216-219.

42. Maret TJ, Collins JP: Ecological origin of morphological diversity: a study of alternative trophic phenotypes in larval salamanders. Evolution 1997, 51:898-905.

43. Pfennig DW: Polyphenism in spadefoot toads as a locally adjusted evolutionarily stable strategy. Evolution 1992, 46:1408-1420.

44. Svanback R, Bolnick DI: Intraspecific competition drives increased resource use diversity within a natural population. Proceedings of the Royal Society B-Biological Sciences 2007, 274:839-844.

45. Bragg AN: Gnomes of the night: the spadefoot toads. Philadelphia, PA University of Pennsylvania Press; 1965.

46. Martin RA, Pfennig DW: Field and experimental evidence that competition and ecological opportunity promote resource polymorphism. Biol J Linn Soc 2010, 100:73-88.

47. Ledón-Rettig CC, Pfennig DW: Emerging model systems in eco-evo-devo: the environmentally responsive spadefoot toad. Evolution and Development 2011, 13:391-400

48. Martin RA, Pfennig DW: Evaluating the targets of selection during character displacement. Evolution 2011, 65:2946-2958.

49. Pomeroy LV: Developmental polymorphism in the tadpoles of the spadefoot toad Scaphiopus multiplicatus. Ph.D. diss. Riverside, CA: University of California; 1981.

50. Pfennig DW: The adaptive significance of an environmentally-cued developmental switch in an anuran tadpole. Oecologia 1990, 85:101-107.

51. Pfennig DW: Cannibalistic tadpoles that pose the greatest threat to kin are most likely to discriminate kin. Proceedings of the Royal Society of London, Series B 1999, 266:57-81.

52. Martin RA, Pfennig DW: Maternal investment influences expression of resource polymorphism in amphibians: implications for the evolution of novel resource-use phenotypes. PLoS One 2010, 5:e9117.

53. Pfennig DW, Rice AM, Martin RA: Ecological opportunity and phenotypic plasticity interact to promote character displacement and species coexistence. Ecology 2006, 87(3):769-779. 
54. Pfennig KS: Female spadefoot toads compromise on mate quality to ensure conspecific matings. Behav Ecol 2000, 11:220-227.

55. Pfennig KS: Population differences in condition-dependent sexual selection may promote divergence in non-sexual traits. Evolutionary Ecology Research 2008, 10:763-773.

56. van Doorn GS, Edelaar P, Weissing FJ: On the origin of species by natural and sexual selection. Science 2009, 326:1704-1707.

57. Lee HJ, Pittlik S, Jones JC, Salzburger W, Barluenga M, Meyer A: Genetic support for random mating between left and right-mouth morphs in the dimorphic scale-eating cichlid fish Perissodus microlepis from Lake Tanganyika. Journal of Fish Biology 2010, 76(8):1940-1957.

58. Raeymaekers JAM, Boisjoly M, Delaire L, Berner D, Räsänen K, Hendry AP: Testing for mating isolation between ecotypes: laboratory experiments with lake, stream and hybrid stickleback. J Evol Biol 2010, 23:2694-2708.

59. Simovich MA: Analysis of a hybrid zone between the spadefoot toads Scaphiopus multiplicatus and Scaphiopus bombifrons. Ph.D. Riverside: University of California; 1985.

60. Gurevitch J, Morrow LL, Wallace A, Walsh JS: A meta-analysis of competition in field experiments. Am Nat 1992, 140:539-572.

61. Dybzinski R, Tilman D: Competition and coexistence in plant communities. In The Princeton guide to ecology. Edited by Levin SA. Princeton: Princeton University Press; 2009:186-195.

62. Kingsolver JG, Pfennig DW: Individual-level selection as a cause of Cope's rule of phyletic size increase. Evolution 2004, 58:1608-1612.

63. Pfennig KS, Pfennig DW: Character displacement as the 'best of a bad situation': fitness trade-offs resulting from selection to minimize resource and mate competition. Evolution 2005, 59:2200-2208.

64. Pfennig DW, Mabry A, Orange D: Environmental causes of correlations between age and size at metamorphosis in Scaphiopus multiplicatus. Ecology 1991, 72:2240-2248.

65. Brodie ED: Correlatonal selection for color pattern and antipredator behavior in the garter snake Thamnophis ordinoides. Evolution 1992 46:1284-1298.

66. Lande R, Arnold SJ: The measurement of selection on correlated characters. Evolution 1983, 37:1220-1226.

67. Stinchcombe JR, Agrawal AF, Hohenlohe PA, Arnold SJ, Blows MW Estimating nonlinear selection gradients using quadratic regression coefficients: double or nothing? Evolution 2008, 62:2435-2440.

68. Mitchell-Olds T, Shaw RG: Regression analysis and natural selection: statistical inference and biological interpretation. Evolution 1987, 41:11491161.

69. Schluter D: Estimating the form of natural selection on a quantitative trait. Evolution 1988, 42:849-861.

70. Simms EL: Examining selection on the multivariate phenotype: plant resistance to herbivores. Evolution 1990, 44:1177-1188.

71. R Development Core Team: R: A language and environment for statistical computing. Vienna: R Foundation for Statistical Computing; http://www.R-project.org/. ISBN 3-900051-07-0.

doi:10.1186/1471-2148-12-136

Cite this article as: Martin and Pfennig: Widespread disruptive selection in the wild is associated with intense resource competition. BMC

Evolutionary Biology 2012 12:136

\section{Submit your next manuscript to BioMed Central and take full advantage of:}

- Convenient online submission

- Thorough peer review

- No space constraints or color figure charges

- Immediate publication on acceptance

- Inclusion in PubMed, CAS, Scopus and Google Scholar

- Research which is freely available for redistribution 